\title{
Representaciones del cambio climático en estudiantes universitarios en España: aportes para la educación y la comunicación ${ }^{1}$
}

\section{Representação das mudanças climáticas em estudantes universitários na Espanha: contribuições à educação e comunicação}

\section{Representations of climactic change among university students in Spain: contributions to education and communication}

\author{
Pablo Ángel Meira-Cartea ${ }^{2}$ \\ Mónica Arto-Blanco ${ }^{2}$
}

\begin{abstract}
RESUMEN
El artículo analiza las representaciones sociales del cambio climático en estudiantes universitarios. El estudio se diseñó atendiendo a la hipótesis de la posible influencia de la formación académica sobre los conocimientos y las creencias de este colectivo. La muestra consta de 284 casos, seleccionados de cuatro titulaciones de grado o licenciatura de la Universidad de Santiago de Compostela (España). Dos titulaciones del campo de las ciencias físico-naturales (Biología e Ingeniería Química) y dos del campo de las ciencias sociales y humanas (Historia y Pedagogía). Los resultados muestran un reconocimiento mayoritario de la causalidad humana en el origen del problema,
\end{abstract}

DOI: $10.1590 / 0104-4060.38041$

1 Este artículo se enmarca en el Proyecto Resclima, financiado por el Ministerio de Economía y Competitividad del Gobierno de España dentro del VI Programa nacional de I+D+i 2008-2011, convocatoria de 2012, REF. EDU2012-33456.

2 Universidade de Santiago de Compostela, Facultade de Ciencias da Educación. Grupo de Investigación en Pedagoxía Social e Educación Ambiental. Santiago de Compostela, Galicia, España. Rúa Xosé María Suárez Núñez, s/n. Campus Vida. C.P.15782. 
así como de las consecuencias y soluciones más destacadas, en consonancia con la ciencia estándar sobre el cambio climático. Se constata, también, una representación social más enfocada a las consecuencias que a las soluciones y la pervivencia de creencias erróneas como la vinculación causal con la capa de ozono y la percepción de que el consenso científico en la materia es bajo. El análisis estadístico solo muestra diferencias significativas en relación a la titulación y el curso para tres de las variables estudiadas. Por último, resultan de especial interés las identidades propias de la cultura académica como condicionantes de las representaciones del cambio climático.

Palabras clave: educación; cambio climático; representaciones sociales; cultura científica.

\title{
RESUMO
}

O artigo analisa as representações sociais das mudanças climáticas em estudantes universitários. $\mathrm{O}$ estudo se desenhou atendendo a hipótese da possível influência da formação acadêmica sobre os conhecimentos e as crenças desse coletivo. A amostra é formada por 284 casos selecionados de quatro titulações de graduação ou licenciatura da Universidade de Santiago de Compostela (Espanha). Duas titulações do campo das ciências físico-naturais (Biologia e Engenharia Química) e duas do campo das ciências sociais e humanas (História e Pedagogia). Os resultados mostram um reconhecimento majoritário da casualidade humana na origem do problema, assim como das consequências e soluções mais destacadas, em consonância com a ciência padrão sobre a mudança climática. Constata-se, também, uma representação social mais enfocada às consequências que às soluções e a permanência de crenças errôneas como a vinculação causal com a camada de ozônio e a percepção de que o consenso científico na matéria é baixo. A análise estatística somente mostra diferenças significativas em relação à titulação e ao curso para três das variáveis estudadas. Por último, resulta de especial interesse as identidades próprias da cultura acadêmica como condicionantes das representações das mudanças climáticas.

Palavras-chave: educação; alterações climáticas; representações sociais; cultura científica.

\begin{abstract}
This article analyses social representations of climactic change among university students. The study was designed to test the hypothesis that the academic field or training may influence students' knowledge and beliefs. The sample consisted of 284 students from four different Bachelor's degree programs at the University of Santiago de Compostela, Spain. Two programs
\end{abstract}


were from the physical-natural sciences (Biology and Chemical Engineering) and two were from the humanities (History and Education). The results showed that a majority of the student sample recognized human causality in the source of the problem, the main consequences and possible solutions. This fits the standard scientific interpretation of climactic change. There was also greater social representation of the consequences rather than the solutions, and a persistence of mistaken beliefs such as a causal connection with the ozone layer and the perception of low scientific consensus on this matter. The statistical analysis only revealed significant differences among degrees and courses for three of the variables examined. Finally, particular identities to the academic culture were of special interest as factors that conditioned representations of climactic change.

Keywords: education; climate change; social representations; scientific culture.

\section{Introducción}

La elección del Cambio Climático (en adelante CC) como objeto de estudio está motivada por la gravedad de las amenazas que proyecta sobre la humanidad y el conjunto de la biosfera. Los informes del Panel Intergubernamental sobre Cambio Climático (IPCC, 2013) han sintetizado los avances científicos de los últimos años, mostrando que la existencia del CC y la responsabilidad humana en su generación son ya constataciones inequívocas. De este modo, la investigación científica ha permitido acotar, conocer y comprender el CC cada vez con mayor certidumbre y precisión.

El CC se ha convertido también en una realidad para la opinión pública internacional (AXA-IPSOS, 2012), traspasando el ámbito académico. El último informe del IPCC (2014) plantea que, ante las valoraciones científicas disponibles, cada vez será más importante conocer las percepciones públicas, así como el modo en que se construyen. Estas percepciones son una variable fundamental para que nuestras sociedades demanden políticas de mitigación y adaptación y participen plenamente en su diseño e implementación (MEIRA-CARTEA, 2008).

La investigación sobre la dimensión social del CC se caracteriza por su plurisiciplinariedad, lo que ha llevado a centrarse en diferentes tópicos. Uno de estos enfoques, en el cual se enmarca esta investigación, aborda el tratamiento del $\mathrm{CC}$ en contextos educativos, explorando los conocimientos de estudiantes y docentes de todos los niveles (KHALID, 2001; PAPADIMITRIOU, 2004; KILINÇ; STANISSTREET; BOYES, 2008; ARTO-BLANCO, 2010; GONZÁLEZ- 
-GAUDIANO; MALDONADO-GONZÁLEZ, 2013). Los resultados obtenidos han servido para elaborar propuestas didácticas que ayuden a que estos colectivos desarrollen una mejor comprensión del problema (SHEPARDSON et al., 2011; PUNTER; OCHANDO-PARDO; GARCÍA, 2010).

Junto a la importancia de las percepciones públicas, el IPCC (2014) manifiesta que las instituciones sociales pueden también contribuir a orientar las acciones individuales hacia la mitigación y la adaptación al CC, fomentando cambios en los estilos de vida. Las instituciones, prosigue el IPCC, han de compartir información y conocimientos para favorecer la acción concertada que permita afrontar los retos comunes. En este sentido, la elección del alumnado universitario como objeto de este estudio está motivada por el papel clave que están llamadas a jugar las universidades en dicho proceso. Se trata de instituciones de referencia en la generación de conocimiento científico y en la formación tanto de profesionales como de ciudadanos y ciudadanas sensibles ante las amenazas del $\mathrm{CC}$ y que se impliquen en la búsqueda de alternativas sociales, económicas y tecnológicas.

\section{Marco teórico}

La presente investigación se desarrolla desde el campo de la educación ambiental, tomando la teoría de las representaciones sociales como referente analítico y explicativo. Para Jodelet (1986), la teoría de las representaciones sociales aborda cómo la representación científica de un fenómeno, en este caso el $\mathrm{CC}$, construida originalmente en un campo de interpretación ajeno al mundo de la vida cotidiana, se objetiva y se ancla en el sistema de representaciones de la cultura común. Esta trasposición se produce, en buena medida, a través de la interlocución de los medios de comunicación y de otros procesos de mediación e interacción social. Dada la necesidad de introducir en el debate público la valoración y las respuestas a los riesgos que comporta la alteración del clima, el conocimiento de las representaciones del CC, y de cómo se están conformando, acaba revelándose como socialmente necesario.

Encarnadas en la cultura común, las representaciones sociales del CC se construyen con elementos (conceptos, teorías, imágenes, etc.) provenientes de la esfera científica, que se combinan con elementos culturales propios de la cultura común (experiencias, tradiciones, creencias, iconografías, identidades, etc.), siguiendo una epistemología distinta a la racionalidad normativa y metodológica que rige la producción del saber científico. En la construcción de este "nuevo" 
objeto intervienen procesos cognitivos individuales y procesos de interacción social esenciales para la socialización de las personas y para la regulación de la vida colectiva (BANCHS, 2000).

La noción de identidad, como concepto que aglutina el componente individual y social, es también un elemento clave para modular la distancia que media entre la representación social y los comportamientos ante el CC (JASPAL; NERLICH; CINNIRELLA, 2014), además de establecer un horizonte moral que permite definir qué es lo relevante (TAYLOR, 1996). La educación universitaria imprime identidad en aquellas personas que la cursan, condicionando su futuro como profesionales y como ciudadanos.

La teoría de las representaciones sociales insiste en que el entendimiento humano y el pensamiento tienen un origen tanto individual como social, y la mayor parte de nuestras interacciones cotidianas se basan en el saber común sin que sea necesario, ni tampoco posible, que el conocimiento científico pueda ocupar todo ese espacio (WAGNER; HAYES; FLORES PALACIOS, 2011). Por ello, el interés no radica en saber si el conocimiento de las personas en relación a un objeto es correcto o no, sino que se busca entender las líneas abiertas por el saber común sobre ese objeto, cómo evoluciona y cuáles pueden ser sus repercusiones valorativas y pragmáticas (SMITH; JOFFE, 2012).

\section{Objetivos y metodología}

En cuanto a los resultados esperados, se parte de la siguiente formulación hipotética: existe un alto grado de homogeneidad en los elementos y las teorías implícitas que articulan las representaciones que comparte el colectivo estudiantil universitario sobre el CC. En este sentido, pensamos que la mayor o menor cercanía disciplinar a las ciencias del clima de la titulación que cursa el alumnado encuestado no influye significativamente en las representaciones sociales que se están generando. Teniendo en cuenta este marco hipotético, los objetivos que orientan esta investigación son:

- Explorar los conocimientos del alumnado universitario sobre las causas, los mecanismos, las consecuencias y las soluciones del CC;

- Determinar si la formación académica de grado superior influye en las representaciones sociales del CC;

- Analizar si existe coincidencia entre los conocimientos sobre el CC proporcionados por el alumnado universitario y las explicaciones científicas en la materia. 
El diseño metodológico está concebido para explorar las percepciones, conocimientos y actitudes ante el $\mathrm{CC}$ de estudiantes universitarios que cursan titulaciones enmarcadas en dos culturas científicas diferentes: del área de las Ciencias, Ingeniería y Arquitectura, por una parte, y titulaciones de Humanidades, Ciencias Sociales y Jurídicas, por otra. La muestra resultante cuenta con 284 estudiantes de la Universidad de Santiago de Compostela (España). La selección se realizó en el curso académico 2011/12, en base a grupos naturales (personas que constituyen una clase), equilibrando proporcionalmente la presencia de estudiantes de cuatro titulaciones de grado o licenciatura (Biología, 22,9\%; Ingeniería Química, 15,5\%; Historia, 26,0\%, y Pedagogía, 35,6\%) y los años de estudio (cursos iniciales, el 53,9\%, o avanzados, el 46,1\%).

Las personas participantes cumplimentaron un cuestionario en el que se optó por combinar preguntas cerradas y abiertas de respuesta múltiple. Con este diseño se pretendió que el alumnado universitario expresara su discurso sobre el CC con mayor libertad, abriendo la posibilidad de que emergieran conceptos, creencias, lógicas interpretativas y temas no captados en otras investigaciones. En contrapartida, el análisis categorial de los datos obtenidos fue más difícil, pero también permitió aflorar dimensiones que pueden ser importantes para personalizar y contextualizar los enfoques educativos y comunicativos sobre el CC en el ámbito universitario.

\section{Resultados y discusión}

Los resultados de la aplicación del cuestionario se desgranan en las tablas y en el cuerpo del texto. El análisis estadístico descriptivo e inferencial al que fueron sometidos atiende a la hipótesis de investigación. Se realizó un análisis de contenido en las preguntas abiertas de respuesta múltiple, seleccionando para este artículo únicamente la primera alternativa de respuesta proporcionada por el alumnado. Por último, se comparan de modo sucinto los datos obtenidos con estudios afines.

\section{Primer pensamiento o imagen al escuchar hablar sobre cambio climático}

Al preguntar "¿Cuál es el primer pensamiento o imagen que te viene a la cabeza cuando escuchas hablar del cambio climático?", la diversidad y dispersión de respuestas llevó a agruparlas en 20 categorías (ver Tabla 1). El deshielo en zonas polares o glaciares $(21,8 \%)$, la contaminación $(15,8 \%)$ y el 
aumento de las temperaturas $(8,8 \%)$ fueron las asociaciones más citadas. Pero, en conjunto, las alusiones a los diferentes efectos del CC alcanzaron el 69,1\%, de modo que el primer pensamiento de los estudiantes sobre el CC se focalizó en las consecuencias y, en menor medida, en las causas (contaminación, el 15,8\%, y falta de conciencia, el 2,8\%). Este patrón se observa también en muestras representativas de población adulta española (MEIRA-CARTEA, 2013), inglesa (SMITH; JOFFE, 2012) o estadounidense (LEISEROWITZ, 2007).

Solo el 1,4\% aludió a una causalidad natural del fenómeno y un testimonial $0,7 \%$ alegó que el CC genera una alarma excesiva o que es preciso más investigación para valorar su importancia, datos muy similares a los recogidos sobre el conjunto de la población española (MEIRA-CARTEA, 2013). Otros elementos destacables, y que coinciden con los estudios demoscópicos ya citados, son las alusiones a la capa de ozono $(3,2 \%)$ y la ausencia de menciones a posibles soluciones o alternativas al problema. Los porcentajes restantes se repartieron entre el 1,8\% que citó fenómenos meteorológicos no vinculados a la alteración del clima, el 7,0\% de respuestas en la categoría otros y el $0,7 \%$ sin respuesta.

\section{Las causas del cambio climático}

Al alumnado se le interrogó específicamente sobre las causas del CC, pidiendo para señalar si pensaban que eran humanas, naturales o una combinación de ambas. Prácticamente ocho de cada diez situó el origen del problema en la actividad humana (principalmente, el 72,9\%, o exclusivamente, el 9,8\%), en consonancia con las tesis centrales del informe del IPCC (2013). El 5,0\% señaló las causas naturales (principalmente, 4,6\%, o exclusivamente, $0,4 \%$ ) y el 7,4\% indicó tanto la causalidad humana como la natural. El 4,9\% de la muestra no respondió. La atribución mayoritaria a causas humanas coincide con los datos obtenidos en estudios sobre el conjunto de la población española (MEIRA-CARTEA, 2013) y sobre muestras de estudiantes universitarios en otros países, como el caso de México (GONZÁLEZ-GAUDIANO; MALDONADO-GONZÁLEZ, 2013).

\section{Los mecanismos causales del cambio climático}

La siguiente cuestión aborda las creencias del alumnado universitario sobre las relaciones que existen entre las emisiones de gases, el efecto invernadero y el deterioro de la capa de ozono. La afirmación "La quema de combustibles fósiles es la principal fuente de emisiones de gases de efecto invernadero" fue considerada probablemente verdadera por el $56,0 \%$ de la muestra y totalmente verdadera por el $33,4 \%$. Nueve de cada diez participantes señalaron como 
correcta la afirmación sin tener presente que los gases de efecto invernadero mayoritarios presentes en la atmósfera derivan de los ciclos del carbono y el nitrógeno. Los combustibles fósiles sí son la fuente principal en cuanto a las emisiones antrópicas (IPCC, 2013).

TABLA 1 - PRIMER PENSAMIENTO O IMAGEN AL ESCUCHAR HABLAR DEL CAMBIO CLIMÁTICO

\begin{tabular}{lc}
\hline Primer pensamiento & Porcentage \\
\hline Deshielo de los polos / glaciares & 21,8 \\
Contaminación & 15,8 \\
Aumento de las temperaturas & 8,8 \\
Destrucción & 7,0 \\
Desertización, desierto y sequía & 6,0 \\
Cambios en el clima & 5,6 \\
Desastres naturales & 3,5 \\
Efecto invernadero & 3,5 \\
Capa de ozono & 3,2 \\
Inundaciones, nivel del mar & 3,2 \\
Falta de conciencia & 2,8 \\
Pérdida de biodiversidad & 2,5 \\
Peligro, problema & 1,8 \\
Fenómenos meteorológicos & 1,8 \\
Fenómeno natural & 1,4 \\
Estaciones & 1,1 \\
Imágenes siniestras & 1,1 \\
Menos alarma, más investigación & 0,7 \\
Futuro & 0,7 \\
Otros & 7,0 \\
No contesta & 0,7 \\
\hline
\end{tabular}

La siguiente afirmación explora si los estudiantes saben que el efecto invernadero es un mecanismo esencial para que la vida en la Tierra sea posible tal y como la conocemos, o si piensan que es una consecuencia negativa de la actividad humana al equiparar el efecto invernadero con el CC. Ante el enunciado "De no ser por el efecto invernadero no existiría la vida tal como la conocemos", el $60,9 \%$ lo valoró correctamente como verdadero (probablemente, 30,3\%; totalmente, $30,6 \%$ ) mientras que el $38,0 \%$ cuestionó su veracidad y el $1,1 \%$ no respondió. El "efecto invernadero", según estos resultados, aparece como una cuestión problemática para un porcentaje relevante de la muestra, coincidiendo con las conclusiones de otras investigaciones realizadas sobre estudiantes de 
educación secundaria (SHEPARDSON et al., 2011) o estudiantes universitarios (KHALID, 2001) en EE.UU (Estados Unidos).

En cuanto a este ítem, hay que indicar que aparecen diferencias estadísticamente significativas $\left(\chi^{2}=17,406, p<.05\right)$ en relación a la variable titulación, siendo el alumnado de Biología el que acumula los porcentajes de respuestas correctas más elevados.

De los tres enunciados sometidos a valoración en esta cuestión, "El agujero de la capa de ozono contribuye al CC" sumó el mayor porcentaje de quienes lo consideraron probablemente $(31,3 \%)$ o totalmente verdadero $(58,8 \%)$, sumando el $90,1 \%$, solo unas décimas más que quienes acreditaron que los gases de efecto invernadero son el resultado de la quema de combustibles fósiles. A pesar de la unanimidad en las respuestas, se trata de una creencia científicamente errónea, ya que el CC y el deterioro de la capa de ozono son problemas diferentes tanto en su etiología como en sus consecuencias.

Según diferentes estudios, la asociación causal entre el deterioro de la capa de ozono y el CC constituye un elemento central en la representación del CC en el marco de la cultura común, destacando la universalidad y la persistencia de esta creencia en grupos de diferentes países, perfiles socio-demográficos y niveles educativos (BOSTROM et al., 1994; KHALID, 2001; SHEPARDSON et al.; PAPADIMITRIU, 2004; ARTO-BLANCO, 2010; MEIRA-CARTEA, 2013; GONZÁLEZ-GAUDIANO; MALDONADO-GONZÁLEZ, 2013).

Otra cuestión que explora las percepciones de los estudiantes sobre los procesos ligados al $\mathrm{CC}$ es la siguiente: “¿Crees que el cambio climático está relacionado con otros problemas?". El 76,8\% indicó que sí existe relación con otros problemas, mientras que el $20,8 \%$ señaló que no y el $2,4 \%$ no respondió. A quienes dieron una respuesta afirmativa, se les preguntó sobre los problemas concretos que asocian con el CC. El 35,3\% señaló diversas cuestiones que se agruparon en la categoría procesos biofísicos, que compila situaciones de deterioro ambiental ligadas a actividades humanas contaminantes. A continuación, el 18,0\% aludió al modelo productivo; la falta de educación, el 12,8\% de las respuestas; y a problemas sociales, el 6,4\%; como categorías que obtuvieron más menciones. La categoría fenómenos catastróficos solo sumó el $0,4 \%$ de las menciones y la categoría otros $(5,0 \%)$ y no contesta $(4,1 \%)$ se repartieron el resto.

La categoría que resulta de especial interés en esta pregunta es la que alude a fenómenos terrestres $(18,0 \%)$, vinculando el CC con fenómenos biofísicos naturales. Las respuestas recopiladas aluden, entre otros, al historial climático de la Tierra, al efecto invernadero o a la influencia solar. A continuación se citan varios ejemplos literales: 
- "La Tierra históricamente sigue ciclos climáticos, es decir, que no tuvo siempre el clima que tiene ahora. Eso sí, la acción humana está acelerando ese proceso" (Ingeniería Química, curso avanzado).

- "En el caso de este último CC el efecto invernadero ya existía, el aumento de los gases solo lo acentúa" (Historia, curso avanzado).

- "De tipo natural, como puede ser el envejecimiento del Sol" (Pedagogía, curso avanzado).

Al comparar las respuestas recopiladas en esta categoría y el tipo de causas del CC indicadas en la segunda cuestión para los mismos casos, destaca que se optó mayoritariamente por señalar principalmente causas humanas. Es decir, las lógicas explicativas que aparecen en la categoría de fenómenos terrestres se vinculan, no con los casos que aluden a una causalidad natural del CC, sino con aquellos que reconocen un peso relativamente importante, pero minoritario, de las causas naturales.

Con respecto a las variables, la prueba chi-cuadrado refleja diferencias estadísticamente significativas en relación a la asociación del CC con los problemas apuntados y el curso académico $\left(\chi^{2}=17,410, p<.05\right)$. El alumnado de cursos iniciales tiende a señalar los procesos biofísicos en mayor medida que el de cursos avanzados, mientras que éste alude en porcentajes más elevados a la vinculación del CC con el modelo productivo y con la falta de educación.

\section{Los efectos del cambio climático}

Los trabajos realizados por el IPCC (2013) apuestan por una visión gradualista del CC a través del diseño de escenarios de emisiones, pero con una alta incertidumbre incorporada por los llamados tipping points o "puntos de ruptura" y por las contingencias de la acción humana. Otros estudios (HANSEN et al., 2007) tienden a destacar la irreversibilidad del proceso de cambio en marcha, con la posibilidad de alteraciones más bruscas, violentas e impredecibles que las sugeridas por los escenarios estándar del IPCC. Condensar en modelos sintéticos los planteamientos científicos sobre el clima (LEISEROWITZ, 2004) y las alteraciones que viene sufriendo es, ante todo, una tarea compleja, pero también muy pertinente para analizar las representaciones sociales y para enfocar la percepción de riesgos en el ámbito educativo. 
TABLA 2 - MODELOS QUE DESCRIBEN LAS CONCEPCIONES DEL CLIMA

\begin{tabular}{lc}
\hline Modelos & Porcentage \\
\hline $\begin{array}{l}\text { El clima cambia de forma lenta. El calentamiento global provocará impactos } \\
\text { peligrosos de forma gradual }\end{array}$ & 36,3 \\
$\begin{array}{l}\text { El clima es estable dentro de unos límites. Si los cambios son pequeños, } \\
\text { el clima recuperará su equilibrio. Si son grandes, se producirán impactos }\end{array}$ & 35,5 \\
$\begin{array}{l}\text { abruptos y catastróficos } \\
\text { El clima tiene un equilibrio delicado. Los cambios pequeños pueden provocar } \\
\text { impactos catastróficos e impredecibles }\end{array}$ & 19,0 \\
$\begin{array}{l}\text { El clima es caprichoso e impredecible. Desconocemos qué puede pasar } \\
\text { El clima es muy estable. El calentamiento global solo tendrá poco o ningún } \\
\text { impacto }\end{array}$ & 3,2 \\
Otros & 0,4 \\
\hline
\end{tabular}

La pregunta "¿Cuál de los cinco modelos siguientes describe mejor tu concepción del clima?” explora las concepciones globales del clima y del CC desarrolladas por el alumnado universitario (Tabla 2). El 36,3\% de la muestra asume que los cambios peligrosos en el clima se producirán de forma gradual, presuponiendo que el clima evoluciona de una forma lenta. El 35,5\% señala como opción preferente que el clima puede recuperar su equilibrio si los cambios son pequeños y que los impactos abruptos y catastróficos solo pueden ser fruto de grandes mutaciones. Es decir, siete de cada diez estudiantes se alinean con una concepción gradualista de la alteración del clima, asumiendo, además, que será posible recuperar el equilibrio climático perdido.

La tercera opción más señalada, con un 19,0\%, argumenta que el clima es un sistema delicado y que incluso pequeños cambios pueden desencadenar procesos catastróficos e impredecibles. El cuarto modelo en número de respuestas, con el 3,2\%, es el que caracteriza el clima como un sistema caprichoso e impredecible, sobre el que es imposible saber qué puede pasar. La última de las opciones de respuesta, aquella que señala que el calentamiento global tendrá pocas repercusiones y que el clima se caracteriza por su estabilidad, acumula un testimonial $0,4 \%$ de las respuestas. El bajo porcentaje de respuestas que sumaron estos dos últimos argumentos muestra el carácter residual del negacionismo climático en la muestra analizada, en consonancia con los resultados obtenidos en preguntas anteriores. El 5,6\% de las respuestas se agrupó en la categoría otros. 
TABLA 3 - PRINCIPALES CONSECUENCIAS EN ESPAÑA DEL CAMBIO CLIMÁTICO

\section{Consecuencias}

Desertización, aridez, escasez de lluvias, sequía

Contaminación (emisiones, $\mathrm{CO}_{2}$, industria)

Aumento del nivel del mar, pérdida de zonas costeras, inundaciones

Aumento de las temperaturas

Cambios en el clima, contraste de temperaturas, desequilibrio

Pérdida de biodiversidad (animales, plantas)

Afectación a la agricultura y la pesca (cambios, pérdidas)

Estaciones (desaparición, cambio)

Lluvia ácida

Pocos cambios

Deforestación

Desastres, catástrofes naturales, fenómenos meteorológicos extremos

Deshielo

Aumento o variación en el régimen de lluvias

Cambios en el ecosistema, en los paisajes o en la naturaleza

Otros

No contesta
Porcentage

25,4

15,5

10,6

6,7

3,2

2,5

2,1

1,0

1,0

1,0

0,7

0,7

0,7

0,4

2,8

3,9

En la siguiente cuestión, al preguntar sobre las consecuencias del CC en España (Tabla 3) las respuestas situaron en primer lugar los efectos relacionados con la desertización, la aridez, la escasez de lluvias y la sequía (25,4\%). Esta familia de consecuencias relacionadas con una menor pluviosidad es también la más reconocida por la opinión pública española (MEIRA-CARTEA, 2013). Después aparecen el aumento de las temperaturas (10,6\%) y la subida del nivel del mar, la pérdida de zonas costeras y las inundaciones (15,5\%). Con porcentajes sensiblemente menores se aluden a la pérdida de biodiversidad $(3,2 \%) \mathrm{y}$ a la afectación a la agricultura y la pesca (2,5\%). Estas consecuencias coinciden, en líneas generales, con los impactos más importantes identificados en la Estrategia Española de Cambio Climático y Energía Limpia (MINISTERIO DE MEDIO AMBIENTE, 2007).

Conviene señalar que las referencias a la contaminación (emisiones, $\mathrm{CO}_{2}$, industria) suman el $21,8 \%$ de las respuestas, pero su presencia puede atribuirse a una comprensión errónea de la pregunta, ya que estas respuestas aluden más a factores causales que a consecuencias propiamente dichas. 
TABLA4 - PRINCIPALES SOLUCIONES PARA LUCHAR CONTRA EL CAMBIO CLIMÁTICO

Modelos

Porcentage

Cambio a energías renovables, menor uso combustibles fósiles, ahorrar energía (electricidad, gas, etc.)

20,1

No contaminar, menos emisiones

17,3

Reflexionar, tomar conciencia, informarse y educación

16,5

Emplear menos el automóvil a favor de alternativas, usar coches menos contaminantes, ir a pie, en bus, etc.

Ley que obligue, medidas, multas, políticas públicas, acuerdos internacionales

Reciclar, reutilizar, reducir residuos sólidos urbanos $\quad 5,3$

Cambio en los estilos de vida (menor consumo y nuevo modelo de producción)

Industrias (reducir contaminación, aplicar impuestos, cierre de centros) $\quad 4,2$

Buen uso de los recursos naturales y sustitución de productos contaminantes

Implicación de todas las personas, trabajo conjunto

No hay solución, demasiado tarde

Reforestación, evitar incendios cuidar espacios naturales $\quad 1,8$

Investigación, estudio $\quad 1,4$

Control del crecimiento de la población $\quad 0,4$

Otros $\quad 1,8$

No contesta 4,2

\section{Las soluciones en la lucha contra el cambio climático}

La principal propuesta del alumnado universitario para enfrentar el CC (Tabla 4) es el cambio a energías renovables, menor uso combustibles fósiles, ahorrar energía (electricidad, gas) (20,1\%), en consonancia con el enfoque del IPCC (2014) en su último informe. Este organismo apuesta por descarbonizar la producción de energía y por la eficiencia energética. La segunda categoría más citada por el alumnado propone de modo genérico y sin especificar cómo, no contaminar, menos emisiones (17,3\%). La tercera opción agrupa propuestas que indican la necesidad de reflexionar, tomar conciencia, informarse $y$ educación (16,5\%), y la cuarta propone emplear menos el automóvil a favor de alternativas, usar coches menos contaminantes, ir a pie, en bus $(7,0 \%)$. La opción de reciclar, reutilizar, reducir residuos sólidos urbanos $(5,3 \%)$ se sitúa en sexto lugar, a diferencia de la relevancia concedida por la población española (MEIRA-CARTEA, 2013). 
El último aspecto que destaca en las respuestas es la ausencia de alusiones a la adaptación al CC, centrándose únicamente en la mitigación. Sin embargo, la adaptación y la mitigación son los dos aspectos fundamentales y complementarios en el planteamiento de las soluciones al CC desarrollado desde instancias internacionales (Organización de las Naciones Unidas - ONU, 1992; IPCC, 2014).

\section{El grado de acuerdo entre la comunidad cientifica}

Para abordar la percepción del alumnado universitario sobre el nivel de consenso dentro de la comunidad científica en torno al CC se formuló la siguiente cuestión: “¿Cuál es tu grado de acuerdo con la siguiente afirmación?: El grado de acuerdo entre la comunidad científica en relación al CC es muy elevado". El $60,2 \%$ de los estudiantes afirmó estar nada $(17,2 \%)$ o poco de acuerdo $(43,0 \%)$ con la afirmación; es decir, seis de cada diez estudiantes, más de la mitad de la muestra, consideran que aún no existe un amplio consenso científico con respecto al CC. El 38,0\% se mostró de acuerdo con la afirmación y el 1,8\% no respondió.

En contraste con esta percepción, como ya se ha destacado, los últimos informes del IPCC ponen de manifiesto el alto nivel de consenso alcanzado en el seno de la comunidad científica, consenso que también se pone de manifiesto en estudios específicos sobre la posición mayoritaria en la comunidad científica que se ocupa del CC (DORAN; ZIMMERMAN, 2009). Sin embargo, la visión social distorsionada que sobredimensiona la falta de consenso científico es común al conjunto de la sociedad española (MEIRA-CARTEA, 2013).

El análisis de variables muestra de forma paradójica que el alumnado de cursos iniciales proporciona el mayor número de respuestas correctas $\left(\chi^{2}=\right.$ $11,483, \mathrm{p}<.05)$, mientras que el de cursos avanzados se muestra más en desacuerdo con la veracidad de la afirmación sobre el consenso científico.

\section{La vinculación del cambio climático con los estudios universitarios}

A la pregunta "¿Crees que tus estudios universitarios están relacionados de alguna forma con el cambio climático?", el 76,8\% de los estudiantes contestó afirmativamente, mientras que el $21,8 \%$ consideraron que no. La categoría otros recogió el $0,3 \%$ de las respuestas y el 1,1\% no contestó. El análisis de variables permite inferir que el alumnado que cursa titulaciones del campo de las Humanidades, Ciencias Sociales y Jurídicas y quienes están en los primeros cursos de su titulación tienden a reconocer en menor medida la vinculación de la formación que reciben con el CC. Sin embargo, las diferencias no son estadísticamente significativas. 
En la segunda parte de la pregunta se pidió a los participantes que especificasen por qué establecían o no la relación. En cuanto a las respuestas afirmativas, el 49,3\% entendió que los contenidos abordados y las materias sí tienen relación, bien porque se han impartido temas ligados con el CC o porque, según la persona que responde, el ámbito científico en el que se forma tiene vinculaciones genéricas con la cuestión. Veamos algunos ejemplos de esta atribución:

- "La Historia puede mostrar cómo procesos como la industrialización influyeron en el aumento de la temperatura... Además, se puede hacer Historia del clima" (Historia, curso avanzado).

- "Porque estudio la ciencia de la vida, Biología, y las investigaciones de la materia se dedican a proponer, para este caso, soluciones al CC" (Biología, curso inicial).

Un porcentaje relevante, el 17,2\%, señaló de forma explícita que su desarrollo profesional futuro estará ligado con el CC o podría orientarse en esa dirección:

- "Como ingeniero químico el desempeño de mi futuro trabajo exige tanto control ambiental de emisiones como uso de combustibles para el funcionamiento de las plantas químicas" (Ingeniería Química, curso avanzado).

- "Porque con nuestra titulación podemos crear programas para que las personas aprendan de alguna forma la importancia de este tema" (Pedagogía, curso inicial).

El 14,1\% de las respuestas explicaron la falta de relación entre el CC y su titulación; el 9,9\% se agruparon en la categoría otros y, por último, el 9,5\% optaron por no responder.

\section{Conclusiones}

La importancia de investigar las representaciones sociales del CC reside, por un lado, en el interés para entender cómo se construye el conocimiento social sobre un objeto científicamente tan complejo y socialmente significativo como 
la alteración antrópica del clima. Y, segundo, dada la inercia del calentamiento global, cada vez es más urgente e inaplazable activar respuestas sociales, políticas, económicas y culturales a esta amenaza, dentro de las que han de ocupar un lugar importante las estrategias educativas y de comunicación orientadas a implicar a la sociedad en dichas respuestas.

La elección para este estudio de alumnado universitario se debe a su mayor afinidad con la cultura científica que otros grupos de población, así como a la relevancia de la institución universitaria como centro educativo y de innovación social. Sin embargo, los resultados obtenidos muestran que los conocimientos del colectivo universitario participante se caracterizan por su homogeneidad, confirmando la hipótesis inicial. El análisis estadístico solo muestra diferencias significativas en relación a la titulación y al curso para tres de las variables estudiadas. Una de ellas indica que el alumnado de Biología reconoce en mayor medida que el efecto invernadero es un fenómeno propio de la Tierra. Las otras dos variables, a pesar de las diferencias significativas, no parecen indiciar necesariamente un mayor peso de la cultura científica relacionada con el CC en los cursos más avanzados o en las titulaciones más afines con las ciencias del clima en función de su campo de estudio.

En líneas generales, los resultados revelan un reconocimiento mayoritario de la causalidad humana en el origen del problema, así como de las consecuencias principales, en consonancia con algunos elementos básicos de la representación científica, pero también con las representaciones sociales del CC que comparte la población española. Se constata una representación social más enfocada a las consecuencias que a las soluciones, en coincidencia con otros estudios. Esto parece indicar una representación del CC con una carga emocional negativa y de tono pesimista, como un problema ante cuyo potencial de amenaza es difícil evocar o visualizar posibles alternativas. Sin embargo, se aprecia un mejor ajuste entre las soluciones propuestas por el colectivo universitario y las aportadas por el IPCC que aquellas que destaca el conjunto de la población española.

Otros elementos a destacar, y que coinciden con los datos obtenidos sobre la representación social del CC en otros colectivos, son la pervivencia de tópicos erróneos como la vinculación causal con el deterioro de la capa de ozono, la creencia en la falta de consenso científico en la materia y el elevado porcentaje de estudiantes que entienden el efecto invernadero como un fenómeno problemático. Estos tópicos indican, por un lado, que la representación del CC sigue muy ligada a la información proporcionada por los medios de comunicación $\mathrm{y}$, por otro, que la incorporación del CC en las aulas es relativamente reciente y puede haberse realizado sin una evaluación previa de los conocimientos y creencias de docentes y alumnado, reproduciendo errores conceptuales ya existentes o alimentando otros. 
La negación o minusvaloración del fenómeno aparece de una forma residual en el conjunto de respuestas. Sin embargo, conviene subrayar que sí existe un porcentaje de respuestas en las que afloran lógicas en las que se equipara el $\mathrm{CC}$ antrópico presente con modificaciones pasadas en la historia climática de la Tierra, o en las que se sobredimensiona la vinculación del CC con otros fenómenos naturales. Los contenidos curriculares, en este sentido, deben ajustarse a los conocimientos científicos más actuales en la materia para evitar alimentar las creencias erróneas o, indirectamente, para evitar contribuir a amortiguar las responsabilidades humanas y a minusvalorar los riesgos asociados al problema.

Además de entender los procesos de construcción del conocimiento social, si el objetivo final es activar la respuesta social ante el CC, puede resultar de especial interés incidir en aquellos aspectos diferenciadores de los grupos sociales. Como se ha recogido en la revisión bibliográfica, las identidades propias de la cultura académica son condicionantes de la representación del CC y pueden servir como un instrumento de comunicación muy eficaz para captar la atención, y lograr una implicación en la lucha contra el CC, tanto en el futuro ámbito laboral del alumnado universitario como en su papel de ciudadanas y ciudadanos.

\section{REFERENCIAS}

ARTO-BLANCO, M. El cambio climático narrado por alumnos de educación primaria y secundaria: propuesta de análisis para dibujos y textos. En: JUNYENT, M.; CANO, L. (Coords.). Investigar para avanzar en educación ambiental. Madrid: Ministerio de Medio Ambiente, Medio Rural y Marino, 2010. p. 11-30.

AXA-IPSOS. Individual perceptions of climate risks. 2012. Disponible en: <http://www. axa.com/lib/axa/uploads/cahiersaxa/Survey-AXA-Ipsos_climate-risks.pdf $>$. Acceso en: $30 / 05 / 2014$.

BANCHS, M. Aproximaciones procesuales y estructurales al estudio de las representaciones sociales. Papers on Social Representation, n. 9, p. 3.1-3.15, 2000.

BOSTROM, A.; GRANGER-MORGAN, M.; FISCHHOFF, B.; READ, D. What Do People Know About Global Climate Change? 1. Mental Models. Risk Analysis, v. 14, n. 6, p. 959-970, 1994.

DORAN, P. T.; ZIMMERMAN, M. K. Examining the Scientific Consensus on Climate Change. Eos, Transactions American Geophysical Union, v. 90, n. 3, p. 22-23, 2009. 
GONZÁLEZ-GAUDIANO, E.; MALADONADO-GONZÁLEZ, A. L. Los jóvenes universitarios y el cambio climático: un estudio de representaciones sociales. Xalapa: Universidad Veracruzana, 2013.

HANSEN, J.; SATO, M.; KHARECHA, P.; RUSSELL, G.; LEA, D. W.; SIDDALL, M. Climate change and trace gases. Philosophical Transactions of the Royal Society A: Mathematical, Physical and Engineering Sciences, n. 365 (1856), p. 1925-1954, 2007.

IPCC. Climate Change 2013: The Physical Science Basis. Contribution of Working Group I to the Fifth Assessment Report of the Intergovernmental Panel on Climate Change [Stocker, T. F., D. Qin, G.-K. Plattner, M. Tignor, S. K. Allen, J. Boschung, A. Nauels, Y. Xia, V. Bex and P. M. Midgley (Eds.).]. Cambridge, United Kingdom and New York, NY, USA: Cambridge University Press, 2013.

. Climate Change 2014, Mitigation of Climate Change. Contribution of Working Group III to the Fifth Assessment Report of the Intergovernmental Panel on Climate Change. [Edenhofer, O., R. Pichs-Madruga, Y. Sokona, E. Farahani, S. Kadner, K. Seyboth, A. Adler, I. Baum, S. Brunner, P. Eickemeier, B. Kriemann, J. Savolainen, S. Schlömer, C. von Stechow, T. Zwickel and J. C. Minx (Eds.).]. Cambridge, United Kingdom and New York, NY, USA: Cambridge University Press, 2014.

JASPAL, R.; NERLICH, B.; CINNIRELLA, M. Human responses to climate change: social representation, identity and socio-psychological action. Environmental Communication, v. 8, n. 1, p. 110-130, 2014.

JODELET, D. La representación social: fenómeno, concepto y teoría. En: MOSCOVICI, S. (Coord.). Psicología social, vol. II. Pensamiento y vida. Psicología social y problemas sociales. Barcelona: Paidós, 1986. p. 469-494.

KHALID, T. Pre-service Teachers' Misconceptions Regarding Three Environmental Issues. Canadian Journal of Environmental Education, n. 6, p. 102-120, 2001.

KILINÇ, A.; STANISSTREET, M.; BOYES, E. Turkish Students' Ideas about Global Warming. International Journal of Environmental \& Science Education, v. 3, n. 2, p. 89-98, 2008.

LEISEROWITZ, A. Before and after The Day After Tomorrow: A U.S. study of climate change risk perception. Environment, v. 46, n. 9, p. 22-37, 2004.

. Communicating the risks of global warming: American risk perceptions, affective images, and interpretive communities. En: MOSER, S. C.; DILLING, L. (Eds.). Creating a climate for change. Communicating climate change and faciliting social change. Cambridge: Cambridge University Press, 2007. p. 44-63.

MEIRA-CARTEA, P. Á. Comunicar el Cambio Climático. Escenario social y líneas de acción. Madrid: Ministerio de Medio Ambiente y Medio Rural y Marino. Organismo de Parques Naturales, 2008.

. (Dir.). La respuesta de la sociedad española antes el cambio climático: 2013. Madrid: Fundación MAPFRE/Aldine Editorial, 2013. 
MINISTERIO DE MEDIO AMBIENTE. Estrategia Española de Cambio Climático y Energía Limpia: Horizonte 2007-2012-2020. Madrid, 2007. Disponible en: <http://www. magrama.gob.es/es/cambioclimatico/publicaciones/documentacion/est_cc_energ_limp_ tcm7-12479.pdf>. Acceso en: 30/05/2014.

ONU. Convención Marco de Naciones Unidas sobre el Cambio Climático. Nueva York, 1992. Disponible en: <http://unfccc.int/resource/docs/convkp/convsp.pdf>. Acceso en: 30/05/2014.

PAPADIMITRIOU, V. Prospective Primary Teachers' Understanding of Climate Change, Greenhouse Effect, and Ozone Layer Depletion. Journal of Science Education and Technology, v. 13, n. 2, p. 299-307, 2004.

PUNTER, P.; OCHANDO-PARDO, M.; GARCIA, J. Spanish secondary school students' notions on the causes and consequences of climate change. International Journal of Science Education, p. 1-18, Sept. 2010.

SHEPARDSON, D. P.; NIYOGI, D.; CHOI, S.; CHARUSOMBAT, U. Students' conceptions about the greenhouse effect, global warming, and climate change. Climatic Change, n. 104, p. 481-507, 2011.

SMITH, N.; JOFFE, H. How the public engages with global warming: A social representations approach. Public Understanding of Science, v. 22, n. 1, p. 16-32, 2012.

TAYLOR, C. Identidad y reconocimiento. Revista Internacional de Filosofía Política, n. 7, p. 10-19, 1996.

WAGNER, W.; HAYES, N.; FLORES PALACIOS, F. El discurso de lo cotidiano y el sentido común: La teoría de las representaciones sociales. Barcelona: Anthropos, 2011.

Texto recebido em 09 de outubro de 2014. Texto aprovado em 13 de outubro de 2014. 
